\title{
Quantification of acute myocardial injury by ShMOLLI T1-Mapping, T2-weighted and late gadolinium imaging in patients presenting with chest pain, positive troponins and non- obstructive coronary arteries
}

\author{
Vanessa M Ferreira ${ }^{1 *}$, Stefan K Piechnik ${ }^{1}$, Erica Dall'Armellina', Theodoros D Karamitsos ${ }^{1}$, Jane M Francis ${ }^{1}$, \\ Matthias G Friedrich², Matthew D Robson', Stefan Neubauer ${ }^{1}$
}

From 2011 SCMR/Euro CMR Joint Scientific Sessions

Nice, France. 3-6 February 2011

\section{Objective}

To assess the ability of ShMOLLI T1-mapping in detecting acute myocardial injury in patients with chest pain, positive troponins and non-obstructive coronary arteries.

\section{Background}

To diagnose acute myocardial injury of varying etiologies, cardiovascular magnetic resonance (CMR) techniques must be sensitive to global and focal changes. ShMOLLI T1-mapping permits quantitative myocardial characterization without contrast agents or reference regions. We compare ShMOLLI T1-mapping against traditional CMR imaging modalities.

\section{Methods}

16 patients ( 8 men, mean age $51 \pm 16$ years) with acute chest pain, Troponin I $>1.0 \mathrm{ug} / \mathrm{L}$ and non-obstructive coronary arteries underwent $1.5 \mathrm{~T}$ CMR imaging within 7 days of presentation (median 3 days). CMR protocol included Shortened Modified Look-Locker inversion recovery (ShMOLLI) for T1-mapping, T2-STIR and late gadolinium enhancement (LGE) imaging. Regional wall motion was assessed with SSFP cines. Volumetric fractions of injured myocardium were quantified by (1) T2 signal intensity (SI) of myocardium:skeletal muscle $>1.9: 1$; (2) T1 $>110 \%$ of normal myocardial T1; and (3) LGE SI $>2 \mathrm{SD}$ of remote myocardium SI.

${ }^{1}$ University of Oxford, Oxford, UK

Full list of author information is available at the end of the article

\section{Results}

8 cases of myocarditis, 4 cases of Takotsubo cardiomyopathy and 4 cases of myocardial infarction were diagnosed based on CMR findings. All demonstrated abnormalities by at least 2 out of the 3 sequences (Table1). All but one (focal myocarditis) demonstrated global/focal increase in T2 SI. All demonstrated global/ focal increase in T1 values. Except for patients with Takotsubo cardiomyopathy, all demonstrated LGE. Volumetric fractions of injury by T2-STIR and T1mapping overlapped but did not necessarily co-localize topographically; there was moderate correlation between the two methods $\left(\mathrm{R}^{2}=0.45\right)$ (Fig. 1 ).

Patients with Takotsubo cardiomyopathy (mean $\mathrm{EF}=54$ $\pm 7 \%)$ had the highest average T1 values $(1028 \pm 34 \mathrm{~ms}$ vs. $968 \pm 76 \mathrm{~ms}$ in normal controls; $\left.\mathrm{p}<0.025^{*}\right)$. Except for one with global myocarditis, patients with myocarditis and MI were without significant LVEF impairment with focal injury. Accordingly, average T1 values in these subgroups were not statistically different from normal.

On a regional level, compared to segments with normal wall motion, segments with abnormal wall motion had significantly increased T1 values $(958 \pm 60 \mathrm{~ms}$ vs. $1068 \pm 76 \mathrm{~ms}$, respectively; $\mathrm{p}<0.001 *$ ) (Fig.2), larger fractions of injury by T1-mapping (median $12 \%$ vs. $62 \%$; $\mathrm{p}<0.001 \%)$, T2-STIR ( $15 \%$ vs. $62 \% ; \mathrm{p}<0.001 \%)$ and LGE ( $8 \%$ vs $10 \% ; \mathrm{p}<0.04 \%$. LVEF correlated best with T1-mapping derived fractions of injured myocardium $\left(R^{2}=0.59\right.$; T2-STIR $R^{2}=0.18$, LGE $\left.R^{2}=0.1\right)$. 
Table 1 Volumetric fraction of acutely injured myocardium

\begin{tabular}{llll}
\hline Patient Groups & $\begin{array}{l}\text { By T2 STIR } \\
(>\mathbf{1 . 9}: \mathbf{1})\end{array}$ & $\begin{array}{l}\text { By T1-mapping } \\
\left(\mathbf{T 1} 1_{\text {Normal }} \times \mathbf{1 1 0} \%\right)\end{array}$ & $\begin{array}{l}\text { By LGE } \\
(>\mathbf{2 S D})\end{array}$ \\
\hline Takotsubo $(n=4)$ & $47 \pm 9 \%$ & $42 \pm 15 \%$ & $10 \pm 5 \%$ \\
Myocarditis $(n=8)$ & $37 \pm 17 \%$ & $34 \pm 27 \%$ & $18 \pm 6 \%$ \\
Myocardial infarct $(n=4)$ & $27 \pm 12 \%$ & $25 \pm 11 \%$ & $25 \pm 10 \%$ \\
All & $37 \pm 16 \%$ & $34 \pm 21 \%$ & $18 \pm 8 \%$ \\
\hline
\end{tabular}

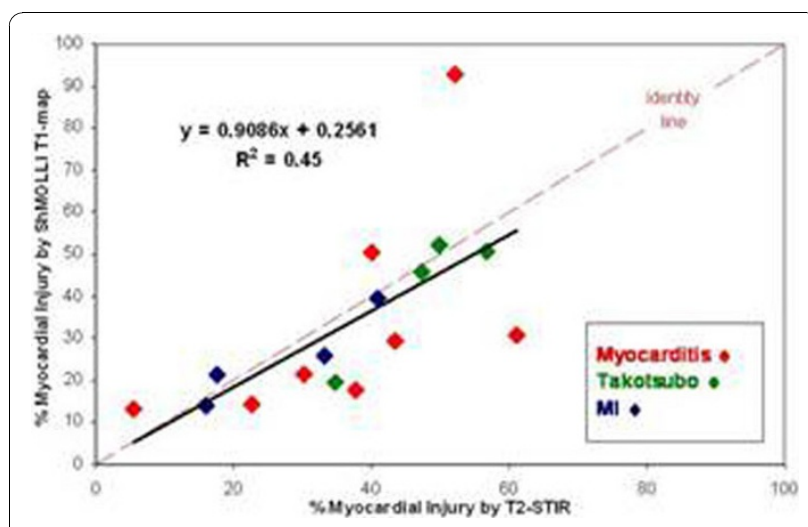

Figure 1 Myocardial injury by ShMolli T-Mapping and T2-STIR.

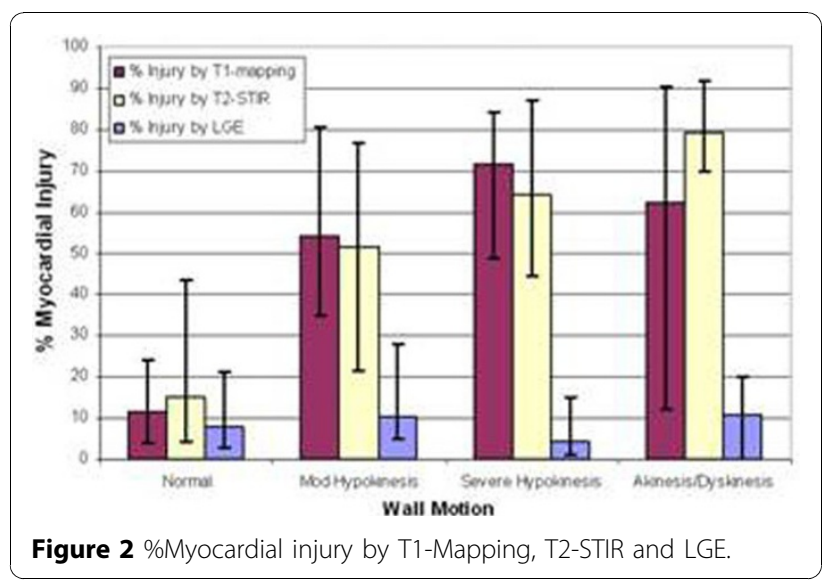

\section{Conclusion}

ShMOLLI cardiac T1-mapping objectively detects acute global and focal myocardial injury of non-ischaemic and ischaemic etiologies. It is a promising method for acute CMR imaging with potential to offer adjunctive information in the characterization of acute myocardial injury.

\section{Author details}

${ }^{1}$ University of Oxford, Oxford, UK. ${ }^{2}$ Stephenson Cardiovascular MR Centre, Libin Cardiovascular Institute of Alberta, Calgary, AB, Canada.

Published: 2 February 2011
doi:10.1186/1532-429X-13-S1-P16

Cite this article as: Ferreira et al: Quantification of acute myocardial injury by ShMOLLI T1-Mapping, T2-weighted and late gadolinium imaging in patients presenting with chest pain, positive troponins and non-obstructive coronary arteries. Journal of Cardiovascular Magnetic Resonance 2011 13(Suppl 1):P16.
Submit your next manuscript to BioMed Central and take full advantage of:

- Convenient online submission

- Thorough peer review

- No space constraints or color figure charges

- Immediate publication on acceptance

- Inclusion in PubMed, CAS, Scopus and Google Scholar

- Research which is freely available for redistribution 